\title{
Perspective
}

PERSPECTIVE Actualité en histoire de l'art

\section{2 | 2010}

\section{Antiquité/Moyen Âge}

\section{Regards sur la peinture antique}

Reflections on painting in Antiquity

\section{Michel E. Fuchs}

\section{(2) OpenEdition}

Journals

Édition électronique

URL : http://journals.openedition.org/perspective/1107

DOI : 10.4000/perspective. 1107

ISSN : 2269-7721

\section{Éditeur}

Institut national d'histoire de l'art

\section{Édition imprimée}

Date de publication : 31 décembre 2010

Pagination : 249-256

ISSN : 1777-7852

\section{Référence électronique}

Michel E. Fuchs, «Regards sur la peinture antique », Perspective [En ligne], 2 | 2010, mis en ligne le 13 août 2013, consulté le 10 décembre 2020. URL : http://journals.openedition.org/perspective/1107 ; DOI : https://doi.org/10.4000/perspective. 1107 


\section{Regards sur la peinture antique}

\author{
Michel E. Fuchs
}

- Ida BALDASSARRE et al., La peinture romaine : de l'époque hellénistique à l'Antiquité tardive, Arles/Milan, Actes Sud/ Motto, 2006. 400 p., 300 fig. en n. et b. et en coul. ISBN : 978-2-74276-216-3; $40 €$.

- Jean-Michel CRoisiLle, La peinture romaine, Paris, Picard, 2005. 376 p., 520 fig. en n. et b. et en coul. ISBN : 978$2708407480 ; 99 €$.

- Jean-Michel CROISILle, Paysages dans la peinture romaine aux origines d'un genre pictural, (Antiqva, 13), Paris, Picard, 2010. 158 p., 181 fig. en n. et b. et en coul. ISBN : 978-270840-865-4; 40,85€.

- Agnès Rouveret, Sandrine Dubel, Valérie NAAS éd., Couleurs et matières dans l'Antiquité, (Études de la littérature ancienne, 17), Paris, Rue d'Ulm, 2006. 304 p., fig. en coul. ISBN : 978-2728803620;19€.

- Gilles SAURON, La Peinture allégorique à Pompéi : Le regard de Cicéron, (Antiqua, 10), Paris, Picard, 2007. 220 p., 133 fig. en n. et b. et en coul. ISBN : 9-782-70840-767-1 ; $59 €$.

- Gilles SAURon, Dans l'intimité des maîtres du monde : les décors privés des Romains, II siècle av. J.-C. - III siècle après J.-C., (Antiqva, 11), Paris, Picard, 2009. 304 p., 230 fig. en n. et b. et en coul. ISBN : 978-2-70840-837-1; $90 €$.

" Le corpus pictural d'époque romaine est vaste et ne cesse de s'accroître, dans toutes les provinces de l'Empire " : voilà comment Jean-Michel Croisille conclut son ouvrage intitulé sobrement La peinture romaine (CROISILLE, 2005, p. 342). Ce n'est pas seulement à la peinture romaine que ce constat doit s'étendre, mais à la peinture antique en général, celle du bassin méditerranéen, du monde grec à celui des Gaulois, en passant par les Étrusques. Les efforts de l'Association internationale pour la peinture murale antique ne sont pas étrangers à l'intérêt porté à ce domaine en pleine expansion ; l'AIPMA, fondée à Cologne en 1989, vient de tenir brillamment ses assises à Ephèse, pour son $\mathrm{XI}^{\mathrm{e}}$ colloque triennal réunissant les spécialistes de la question ${ }^{1}$. En outre, l'inlassable activité du Centre d'étude de peintures murales romaines a joué et joue encore un rôle majeur dans la promotion de l'étude, de la conservation et de la restauration des peintures murales, révélant la part souvent ingrate de l'inévitable état fragmentaire des témoins découverts dans les provinces romaines. Créé par Alix Barbet, directrice de recherches honoraire du CNRS, le CEPMR poursuit ses activités depuis Paris ou Soissons et fait référence pour nombre de chercheurs en peinture murale - de "picturalistes " aimerait-on dire - des anciennes et des nouvelles générations. La publication de La peinture murale en Gaule romaine de Barbet et la réédition de sa Peinture murale romaine: les styles décoratifs pompéiens ${ }^{2}$, augmentée de chapitres conclusifs renvoyant aux nouvelles découvertes et publications, démontrent la grande activité qui se manifeste autour d'un art toujours à la recherche de reconnaissance, du moins en dehors de Rome et de Pompéi.

Art ou artisanat ? La question a très tôt été posée à propos de la peinture murale antique. On se souvient de l'avis cinglant de Stendhal à se sujet : "Ces peintures, si considérables aux yeux des érudits, sont des fresques enlevées à Pompeia et à Herculanum. Il n'y a point de clair obscur, peu de coloris, assez de dessin et beaucoup de facilité... Cela ressemble à des mauvais tableaux du Dominiquin... Il faut être sot comme un savant pour prétendre que cela est supérieur au XV $\mathrm{XV}^{\mathrm{e}}$ siècle : ça n'est qu'extrêmement curieux" (Naples, 5 mars 1817) ${ }^{3}$. Le nombre des découvertes récentes et la reprise régulière des documents campaniens, qu'ils soient d'Herculanum, de Pompéi, de Stabies ou des villas alentour, invitent aujourd'hui plusieurs spécialistes à exprimer leurs idées sur le sujet. La tendance générale est à la prise en compte du contexte aussi bien architectural et fonctionnel que social dans lequel une peinture murale voit le jour. Grâce à l'affinement des méthodes de datation, avec l'augmentation du nombre de rapprochements possibles, la question chronologique, bien que primordiale, n'est plus un enjeu aussi prégnant que par le passé. C'est aussi la part des études iconographiques qui s'est relativement réduite, ou plus précisément l'approche interprétative qui était celle de grands noms comme Ranuccio Bianchi Bandinelli, Hendrik Gerard Beyen, Franz Cumont ou Karl Schefold. Ces savants sont d'ailleurs souvent cités dans les publications ici recensées, qui comptent, elles aussi, parmi les recherches qui vont au-delà du descriptif. 
Considérer la date des témoignages retenus comme l'un des éléments permettant d'en approfondir le sens, sans en faire un but ultime, voilà ce qu'heureusement proposent nos auteurs.

\section{La diffusion de modèles}

Sans lui porter une attention exacerbée, la question chronologique s'avère toujours nécessaire pour mieux saisir les différentes implications d'un décor dans la société qui l'a vu naître. Dans La Peinture romaine : de l'époque hellénistique à l'Antiquité tardive (BALDASSARRE et al., 2006), publié en grand format dans un premier temps et dans un ouvrage au format de poche très pratique dans son ultime version française, les auteurs réunis autour d'Ida Baldassarre, responsable de la précieuse encyclopédie des peintures et des mosaïques de Pompéi ${ }^{4}$, ont replacé la peinture romaine dans le cours de l'histoire, en insistant sur l'apport contextuel indispensable à sa compréhension. L'ouvrage met en lumière le pas décisif apporté par les récentes découvertes de tombeaux peints en Macédoine ; jusqu'alors, ce que les textes anciens mentionnaient des grands peintres de l'époque d'Alexandre le Grand et de ses successeurs n'était perceptible que grâce à l'écho qu'on en avait par le biais des peintures plus tardives, particulièrement celles de la région vésuvienne. De tels modèles macédoniens, adaptés selon les réalités locales, ont inspiré les monuments recensés dans le bassin méditerranéen, appartenant à une aristocratie qui s'entourait de peintres de qualité. Ainsi, en Italie centrale et méridionale, on voit s'élaborer dès la fin du $\mathrm{IV}^{\mathrm{e}}$ siècle avant J.-C. un langage mixte entre traditions italiques (campanienne, étrusque et lucanienne) et effervescence grecque colportée par les cités de Naples ou de Tarente. C'est dans ce contexte que naît la peinture romaine. Le constat porte pour beaucoup sur la peinture fu-

1. Rome, Palatin, Maison d'Auguste, salle des masques : fronts de scènes ornés de masques et rehaussés de sanctuaires rustiques [SAURON, 2007, fig. 130]. néraire, mais la peinture domestique n'est pas en reste : c'est dans d'autres régions qu'il faut chercher les relais qui permettent d'aboutir aux premiers témoignages d'intérieurs peints romains, dans les maisons de Pella et d'Amphipolis en Macédoine, dans celles de Kertch/Panticapée en Crimée, d'Arpi en Italie du Sud ou de Gela et de Morgantina en Sicile. À l'aune de ces exemples, le "premier style " pompéien, selon la typologie élaborée par August Mau à fin XIX ${ }^{\mathrm{e}}$ siècle et fondée sur le chapitre V du livre VII de Vitruve, se révèle être une variante régionale du style structural hellénistique répandu à travers toute la Méditerranée dès le IV ${ }^{e}$ siècle avant J.-C.

Si la région du Vésuve du $\mathrm{I}^{\mathrm{er}}$ siècle avant J.-C. fait indéniablement référence pour les chercheurs - elle qui a muséifié si précieusement des séries d'exemples qui ouvrent le discours à une finesse d'analyse rarement atteinte ailleurs -, l'apport de régions autres que celles de Rome et de la Campanie se fait peu à peu entendre grâce à des ensembles datés comme ceux de Glanum/Saint-Rémy-de-Provence en Gaule Narbonnaise ou du Magdalensberg en Autriche. Au début de l'Empire, on assiste en effet au développement d'un art de cour que les témoignages archéologiques nous donnent l'occasion d'appréhender grâce à la découverte de plusieurs demeures d'Auguste et de ses proches, un art qui servit de modèle aussi bien stylistique que comportemental, comme l'illustrent les peintures campaniennes et les décors d'autres provinces (fig. 1). Le mouvement lancé par Auguste fut suivi, dans son principe, jusqu'à la fin de l'Antiquité romaine. Toute la difficulté est aujourd'hui d'en saisir les nuances après la destruction de Pompéi et de sa région en 79 après J.-C., faute d'un site de référence équivalent pour les périodes postérieures. Il est significatif à cet égard de constater chez Baldassarre et ses collègues comme dans le manuel de Croisille que les peintures de la fin du $\mathrm{I}^{\mathrm{er}}$ siècle au $\mathrm{IV}^{\mathrm{e}}$ siècle après J.-C. font l'objet d'une présentation et d'une analyse moins fournies que pour les périodes précédentes. Elles sont même dites "tardives" par Croisille lorsqu'il envisage la période qui va de Domitien aux Sévères,

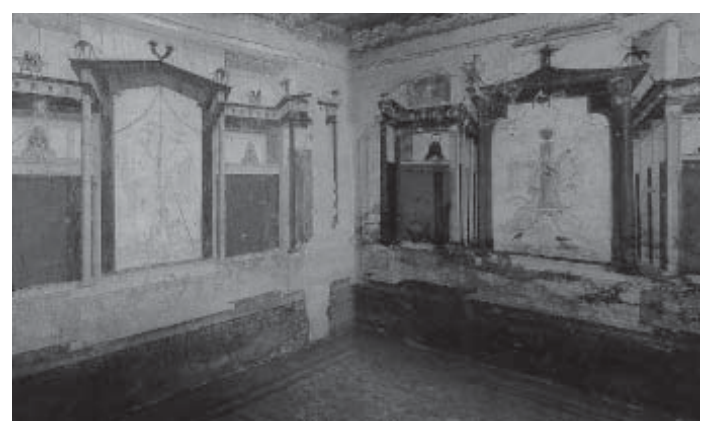


soulignant la difficulté d'en faire la synthèse à partir d'une documentation disparate et fragmentaire (CROISILLE, 2005, p. 103). Rome et Ostie ont pourtant livré nombre d'exemples dignes de la Paix romaine; des ensembles cohérents témoignent de la réalisation de programmes à Ephèse, à Sabratha ou à Narbonne ; tombeaux et catacombes ne sont pas en reste à l'Orient et à l'Occident de l'Empire ; et même le décor des pièces secondaires a déjà suscité une recherche aux conclusions non négligeables, tout particulièrement pour ce qui concerne le style dit linéaire propre au III $^{\text {e }}$ siècle ${ }^{5}$. Quant aux questions que soulève le plafond constantinien de Trèves, malgré sa fragmentation, celuici n'a rien à envier au décor augustéen de l'Aula Isiaca sur le Palatin à Rome.

\section{Des décors typés}

Reconnaissons-le toutefois, la peinture pompéienne bénéficie d'une tradition de recherche et de conservation de vastes surfaces peintes auxquelles la plupart des autres ensembles ne peuvent prétendre. C'est ainsi que des études sont consacrées à des périodes très précises de l'histoire de l'art romain, à l'instar de celle de Gilles Sauron, La peinture allégorique à Pompéi : le regard de Cicéron (SAURON, 2007), dans laquelle il ausculte le contexte d'élaboration des peintures dites mégalographiques, autrement dit composées de personnages grandeur nature intégrés à des scènes créées du temps des Cicéron, Pompée ou César. Fasciné par les décors de la fin de la République, spécialement par ceux qui composent le cadre de vie de l'aristocratie romaine, Sauron pointe les contradictions de la recherche à leur égard et engage à poursuivre leur analyse détaillée pour viser une interprétation qui écarte les hypothèses fragiles et infondées. Il en arrive au principe de l'allégorie picturale comme concept propre à une période bien précise, qu'il situe entre les années 80 et 40 avant J.-C. (SAURON, 2007, p. 196-197). En reprenant la définition qu'en fait le rhéteur Quintilien dans son Institution oratoire (VIII, 6, 44), il définit l'allégorie comme l'expression de ce qui "présente un sens autre que celui des mots (des images dans notre cas), et parfois contraire ". Elle se caractérise par une suite de métaphores, l'exposé du contraire de ce que l'on dit ou l'atténuation de propos fâcheux. Sauron montre que ce principe de l'allégorie est adopté dans chacun des exemples marquants du $\mathrm{II}^{\mathrm{e}}$ style pompéien, à commencer par la célèbre frise de

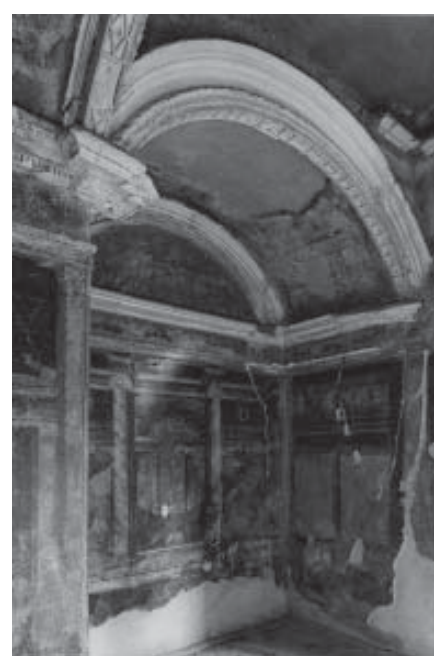
la Villa des Mystères àPompéi, mais aussi à Boscoreale, dans la villa dite de P. Fannius Synistor - tout au moins dans sa dernière phase d'occupation - ou à Oplontis, dans la villa dite de Poppée (fig. 2). Son analyse aboutit à la révision de l'identité du propriétaire - et commanditaire du décor pour chacune de ces villas à un moment de leur occupation. Ainsi, les portiques et la palestre de la villa de Boscoreale, du milieu du I ${ }^{\text {er }}$ siècle avant J.-C., reviennent à Publius Aninius, duumvir de Pompéi, qui s'inspira des thermes de Stabies qu'il avait fait restaurer. Quant à Oplontis, son riche décor est très vraisemblablement dû au consul de 61 avant J.-C., M. Pupius Piso, érudit ami de jeunesse de Cicéron.

À en croire Sauron, l'allégorie picturale serait exclusivement liée à l'aristocratie romaine et au $\mathrm{II}^{\mathrm{e}}$ style dans sa phase initiale ; elle mourrait définitivement avec le passage au III $^{\text {e }}$ style sous Auguste. Ce n'est naturellement pas le changement de style qui en est la cause, mais bien la volonté du premier empereur d'adopter une nouvelle approche, à l'aide d'une peinture qui rende compte à la fois de l'annexion de l'Égypte et donc de l'intégration à Rome de l'empire d'Alexandre le Grand, et du retour à l'Âge d'or sous le signe d'Apollon. Dans cet ordre immuable, rendu possible grâce à l'autorité impériale, l'allégorie au service des grands aristocrates de la fin de la République, elle qui reflétait la défense de leurs prérogatives dans un monde perpétuellement troublé par les guerres intestines, n'a plus droit de cité. Est-ce à dire que l'allégorie sera véritablement 
écartée du discours de l'élite romaine jusqu'à l'Antiquité tardive ? Dans son ouvrage Dans l'intimité des maîtres $d u$ monde: les décors privés des Romains (SAURON, 2009), Sauron n'est plus aussi tranchant qu'en 2007 : même si le II ${ }^{\mathrm{e}}$ style initial reste pour lui le lieu privilégié de ce discours, celui-ci est transposé au $\mathrm{I}^{\text {er }}$ siècle après J.-C. dans le domaine des tableaux peints au milieu d'architectures feintes ou plus significativement dans des cycles statuaires comme le célèbre groupe du Laocoon ou celui d'Ulysse et du Cyclope dans la grotte de Sperlonga.

Dans cette optique, le III $^{\text {e }}$ style n'est pas en rupture avec le $\mathrm{II}^{\mathrm{e}}$ style ; il en perpétue les thèmes, mais dans des schèmes différents, renouvelés, repris, vivifiés sous Auguste et sous les empereurs julio-claudiens, comme l'explique Agnès Rouveret (BALDASSARRE et al. 2006, P. 130-165). Il atténue dans un premier temps les effets d'architectures pour laisser place à des panneaux rouges, noirs et blancs. L'étalage de ces couleurs marque-t-il véritablement la fermeture de la paroi, comme on l'a dit souvent pour le III $^{\mathrm{e}}$ style ? Ou ne faudrait-il pas rapprocher l'usage du rouge de la pourpre impériale ou du printemps, comme le souligne Jean Trinquier lorsqu'il traite des couleurs du paysage dans la littérature latine. Cet auteur précise que le rouge est la couleur par excellence dans le domaine de la teinturerie (ROUVERET, DUBEL, NAAS, 2006, p. 213-259, en particulier p. 239241 pour l'usage du rouge), le blanc rappelant le marbre et marquant l'éternité, le noir évoquant le luxe d'un bronze de Corinthe. En effet, la patine sombre des bronzes était particulièrement recherchée à la fin de la République et l'alliage que l'on appelait "bronze de Corinthe " permettait des nuances allant du noir profond au noir pourpre ou au noir bleuté, selon les quantités respectives d'or, d'argent, d'arsenic, de fer et d'étain. Pline l'Ancien alla jusqu'à dire dans son Histoire Naturelle (XXXIV, 1) que ce bronze fascinant venait " avant l'argent, et presque même avant l'or " (Sophie DescampsLequime dans Rouveret, DUBEL, NAAS, 2006, p. 79-92, surtout p. 85-88).

Le nouvel angle de vue initié par les peintures de $\mathrm{II}^{\mathrm{e}}$ style conduit à nuancer le dernier type de décor pompéien, le IV e style : c'est du modèle qu'il faut partir, accessible soit par la littérature, soit par l'archéologie. Si le changement de style se dessine sous l'empereur Claude, c'est à Néron que l'on doit un témoin extrêmement opérant, celui de la Domus Aurea à Rome, que l'on mettra en résonance avec le Satyricon de Pétrone (CROISILle, 2010, p. 8688). La thématique du paysage est ici emblématique de l'évolution romaine d'un genre pictural : celui-ci fait la synthèse d'éléments naturalistes pour former des tableaux que l'on dira paysagers et que l'on appelait topia, un nom certes grec, mais qui a été utilisé pour la première fois dans des textes latins (fig. 3). Selon Croisille, le paysage est une invention romaine et, à ce titre, il reflète la politique et l'idéologie de l'Empire (CROISILle, 2010, p. 137-140). Sous Auguste, le sacro-idyllique l'emporte dans un style proche des bucoliques de Virgile ou des épodes d'Horace. Sous Claude et Néron, la piété augustéenne cède la place à une artialisation que l'époque flavienne tend à diffuser tout en laissant apparaître la marque des puissances supérieures régnant sur la nature et le destin des hommes. Cet art consommé de la nature reconstruite est particulièrement visible dans les paysages de viridaria du IV style, où s'affrontent des animaux comme dans les venationes décrites par Martial à l'occasion de l'inauguration du Colisée, puis sous Domitien (Livre des spectacles et Epigrammes I, 48, 51, 60...).

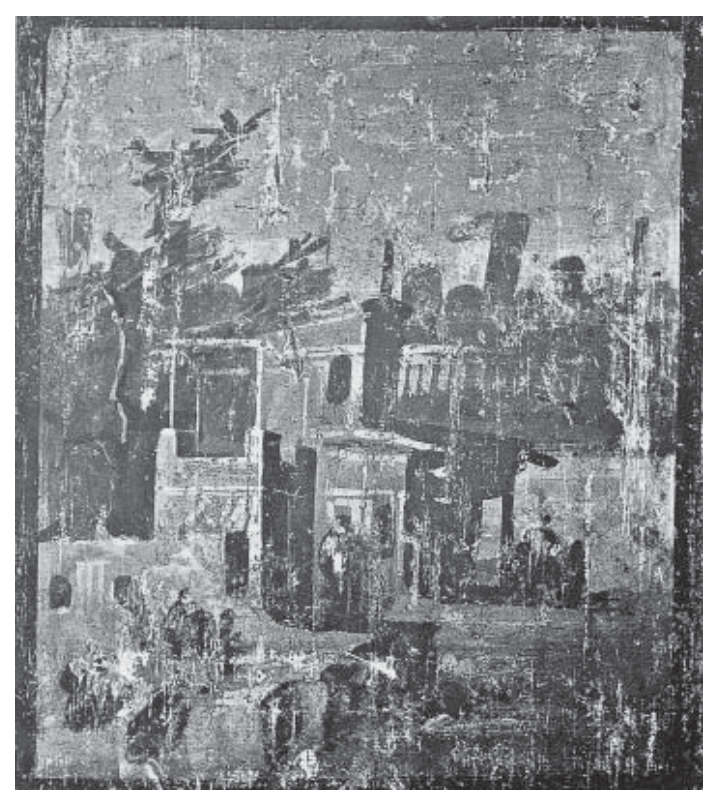


Pour les périodes flavienne et suivante, à l'égal de la chronologie des décors, la typologie et l'interprétation des peintures sont abordées avec grande prudence par Croisille et de manière plus hardie par Ida Baldassarre et Monica Salvadori. Le premier avance dans les pas d'un Karl Schefold ${ }^{6}$ pour la fin du I $^{\text {er }}$ siècle après J.-C., reprenant lui-même d'autres chercheurs pour approcher les Imagines de Philostrate, cette galerie de tableaux décrite sous les Sévères (CROISILle, 2005, p. 334-337). Les secondes, fortes de leurs connaissances des peintures d'Ostie et des provinces, parlent d'une déstructuration du système architectural sous Trajan et Hadrien à Rome et à Ostie, là où nous verrions au contraire un allègement de l'architecture dans un esprit de retour aux valeurs augustéennes du III $^{\mathrm{e}}$ style, tout en laissant se développer des effets de façades théâtrales (BALDASSARRE et al., 2006, p. 279-297). Si le marbre et son imitation peinte expriment le luxe dans les maisons comme dans les tombes tout au long du $\mathrm{II}^{\mathrm{e}}$ siècle et dans les siècles suivants, la peinture des mythes revient en force dans le domaine funéraire. Le III $^{\mathrm{e}}$ siècle serait plutôt celui du décoratif alors que le IV siècle montre un évident retour à un certain classicisme, selon Baldassarre et Salvadori. Très bref sur la peinture $\mathrm{du} \mathrm{IV}^{\mathrm{e}}$ siècle et sous l'autorité chrétienne, Croisille livre cette conclusion qui sonne comme un credo : soumettant tout à la glorification de son dieu, le christianisme est à l'origine de « la disparition de l'art pictural proprement romain " (CROISILLE, 2005, p. 339).

\section{Matériaux et techniques}

Si l'école française menée par Barbet a eu le mérite de mettre très tôt en évidence l'approche technique de la peinture murale romaine, par l'étude des empreintes de support, des mortiers, des tracés préparatoires et du traitement des surfaces peintes, les auteurs recensés, à l'exception de Croisille dans son manuel de 2005 , ne l'évoquent que brièvement ou pas du tout. Pour Baldassarre et ses collègues, le choix des peintures présentées a été fait en fonction de leur aspect représentatif d'une époque et d'un style, en soulignant leurs caractéristiques chromatiques et constructives. La technicité et l'élaboration poussée des schémas figurés provenant de Grande Grèce sont mentionnées comme faisant le lien avec Rome et le monde étrusco-italique ; c'est cependant le langage pictural développé par la suite qui leur importe, intégrant et transformant les réalités locales selon un processus qui sera suivi par le voisin conquérant. Notons que les résultats d'analyses archéométriques récentes ${ }^{7}$ permettent pourtant de nuancer certains propos, en particulier pour ce qui concerne le rouge cinabre qui n'est pas ce pigment que l'on trouve essentiellement entre 20 avant et le milieu du ${ }^{\text {er }}$ siècle après J.-C., avant qu'il ne se raréfie : il est en usage, de manière diversifiée, au moins jusqu'au III $^{\mathrm{e}}$ siècle. Rappelons aussi que les résultats d'analyses ne sont pas nécessairement révélateurs d'un état de fait. Si des laques organiques n'ont pas été identifiées sur les pigments analysés en Suisse, cela n'indique pas qu'elles sont inexistantes, mais que les méthodes utilisées n'ont pas permis d'en faire le constat.

De son côté, malgré son titre, l'ouvrage Couleurs et matières dans l'Antiquité ne s'attarde pas sur l'apport des analyses physico-chimiques et sur l'observation technique des peintures murales. Seul un article traitant de la polychromie des bronzes intègre l'analyse de composants pour mieux mettre en rapport les constats actuels avec les textes antiques; la reproduction de recettes antiques, par exemple, vient confirmer les observations sur le bronze noir de Corinthe faites par l'alchimiste Zosime, qui vivait en Egypte au $\mathrm{III}^{\mathrm{e}}$ siècle après J.-C. (Descamps-Lequime dans ROUVERET, DUBEL, NAAS, 2006, p. 86-87)

\section{La couleur et le texte}

L'un des apports capitaux à l'histoire de la peinture murale antique tient, ces dernières années, à la mise au jour de tombes et de maisons macédoniennes, au jeu sur les couleurs et à la qualité picturale qu'elles manifestent. Que ce soit à Vergina, à Aghios Athanassios près de Salonique, à Aineia ou à Lefkadia, les exemples ne manquent pas pour enfin démontrer que la peinture murale du monde grec ne se limitait pas, loin s'en faut, au masonry style, le style structural connu jusqu'ici. En Grande Grèce, de nouveaux témoins sont venus compléter ceux 
des tombes de Paestum, à Nola, Tarente et Arpi, ainsi qu'à Naples et à Capoue. Ces découvertes, alliées à l'approfondissement de l'étude des décors eux-mêmes, ont poussé les chercheurs à ne plus faire de la peinture la seule illustration des textes antiques, mais bien à mettre en évidence l'interaction entre les deux supports. En tant que latiniste et traducteur de Schefold, à la mémoire duquel il dédie d'ailleurs son livre de 2005, Croisille insiste sur le lien qui doit être établi entre art littéraire et art pictural, tous deux miroir d'une société, de son évolution et de ses préoccupations.

Dans Couleurs et matières dans l'Antiquité, ouvrage universitaire issu d'un colloque, douze études se concentrent sur les couleurs de la peinture d'une part, et sur le jeu des couleurs et des matières d'autre part. "Tout philosophe voit rouge quand il entend parler de couleur " disait Goethe. Le discours n'a pourtant jamais été interrompu sur ce sujet et, comme le rappelle Agnès Rouveret dans son excellente introduction, les nouvelles découvertes et les progrès scientifiques faits à propos de la vision, et l'analyse des pigments utilisés invitent à retourner aux textes antiques pour une lecture croisée.

Le choix du mot pour dire la couleur révèle une culture à une période donnée. Les médecins hippocratiques ne décèlent-ils pas les symptômes d'une maladie dans les variations des couleurs de l'épiderme, alors qu'en grec les mots chroa, "peau ", et chrôma, "couleur", ont la même étymologie ? La couleur chair, l'andreikelon, est aussi au centre des préoccupations des peintres du IV ${ }^{\mathrm{e}}$ siècle avant J.-C. et les sculpteurs ont rivalisé d'inventivité pour rendre l'éclat du vivant à leurs statues de bronze ou de marbre. Les recherches de l'époque romaine sur les couleurs laissèrent leur empreinte sur le goût antique. Le noir des bronzes de l'épave de Mahdia, par exemple, une patine obtenue à l'aide de sulfure de cuivre qui entre dans la

4. Rome, Villa de la Farnésine, triclinium C: paysages et scènes de jugement sur paroi à fond noir [SAURON, 2009, fig. 123]. composition du fameux bronze de Corinthe, signale la primauté, à la fin de l'époque hellénistique, du contraste entre le blanc et le noir sur le clair-obscur classique. Il marque ainsi un engouement pour la couleur décorative dont le triclinium noir de la Farnésine sous Auguste (fig. 4) ou l'usage de marbres polychromes dans l'Empire sont des exemples manifestes. Si la couleur joue un rôle important dans la poésie, elle n'est pas nécessairement reportée de la même manière en peinture. À cet égard, la comparaison entre paysages littéraires et paysages peints est des plus parlantes : dans la première, la prairie en fleurs, polychrome, est un motif récurrent, alors qu'il n'apparaît que rarement dans la seconde, puisque réservé à la peinture de jardin. N'est-ce pas dû toutefois à un changement ou à l'évolution des codes expressifs ? L'étude du mot color chez les théoriciens de la rhétorique montre qu'il est d'abord lié à la forme du discours chez Cicéron, avant de définir le contenu même du discours chez Pline l'Ancien. Les couleurs seront d'ailleurs pour l'encyclopédiste un critère de choix pour la classification des matériaux. En peinture et en littérature antique, le mot du début comme celui de la fin revient à Homère, le visionnaire qui, grâce à sa phantasia, a donné un modèle à toute ekphrasis, à toute peinture par sa description du bouclier d'Achille.

Fidèle à la tradition homérique, et par la suite à la tradition classique et hellénistique, l'art romain serait-il donc essentiellement citation, copie, pastiche des modèles grecs ? À la tendance trop longtemps suivie de chercher toujours derrière le tableau romain l'original grec, Croisille répond avec nuance, montrant toutes les difficultés qu'il y a à reconnaître tel peintre derrière telle œuvre. Il insiste au contraire sur les choix proprement romains dans le domaine $\mathrm{du}$ paysage, de la nature morte, du portrait et même des scènes de genre. À ces ouvres peut être tout particulière ment attribué le terme de compendiaire, compendiaria (via), dont Pétrone et Pline se font l'écho, signe d'une dégénérescence de la

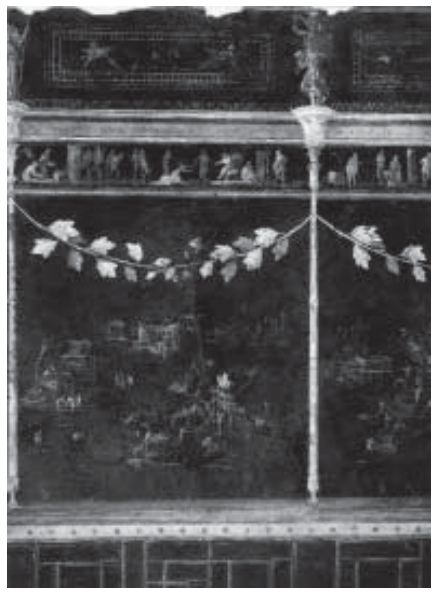


peinture pour le premier ; largement diffusé sous l'Empire, le procédé compendiaire, "abrégé ", privilégie les contrastes lumineux et colorés aux dépens du dessin, dans un traitement la plupart du temps rapide, qui n'empêche d'ailleurs pas un certain talent (CROISILlE, 2005, p. 281 et 303).

\section{Un discours en images}

La découverte, au nord de la Grèce, de tombes princières de la fin du IV siècle avant J.-C. permet d'interroger l'importance du commanditaire et des mécanismes idéologiques pour la réalisation d'une œuvre murale. Cette préoccupation est largement développée par Sauron à propos des peintures de la fin de la République romaine (SAURON, 2007 et 2009). Le jeu sur la perception subjective du spectateur antique est au centre de son discours, de même que la recherche de codages secrets renvoyant à l'usage de l'allégorie par l'aristocratie du I $^{\mathrm{er}}$ siècle avant J.-C. La quête du sens se fait alors dans l'omniprésence du théâtre, les peintres agissant comme des scénographes, maîtres de la représentation d'architectures vues en perspective. Les commanditaires sont fascinés par le pouvoir des images plus que par la virtuosité technique, davantage caractéristique de l'approche grecque. Leur langage, celui des dieux, s'exprime sur les murs. Comment dès lors retrouver le premier regard posé sur un décor ? La solution se cache dans un savant mélange de littérature et d'iconographie, sans hésiter à tenir compte du mauvais goût de l'atrium de Trimalchion chez Pétrone (Satyricon XXIX). Le supplément de sens est donné par la philosophie du temps, les transports pythagoriciens, le serein épicurisme. Le décor statuaire de la Villa des Papyrus à Herculanum, c'est le paysage d'Epicure reconstitué, le jardin des Bienheureux à demeure sous la République ; les parois peintes de la Maison des Vettii à Pompéi, ce sont les rêveries d'un bourgeois sous l'Empire.

Traitant de paysages, Rouveret a souligné à quel point le monde gréco-romain était une société paysagère, mettant aussi bien en scène des fantaisies imaginaires que collectionnant des mirabilia ${ }^{8}$. Dans l'iconographie romaine, cependant, la recherche formelle suscite moins d'intérêt qu'à l'époque hellénistique ; à la différence de la littérature, on privilégie la répétition de formules stéréotypées. Sauron cherche, quant à lui, à retrouver le regard que l'on portait sur les tableaux, sur un décor choisi

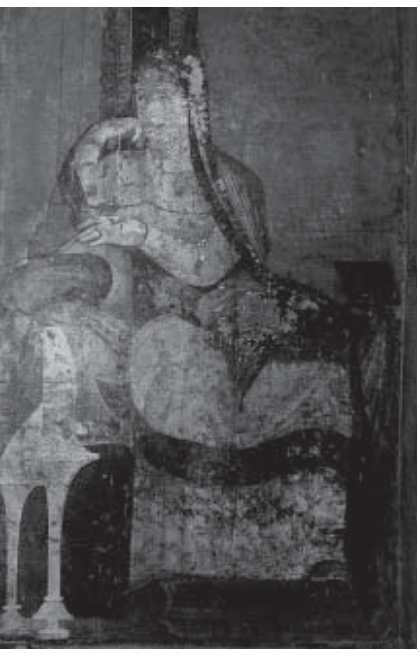

5. Pompéi, Villa des Mystères, salle 5 ou " des Mystères ", paroi ouest : la Domina pensant à sa vie [SAURON, 2009, fig. 112]. dans un contexte donné. Il en tire un lexique, aux notions fortement résumées ici : tout commence par la certitude, le certus de l'identifiable. Suit le goût de l'énigme, de l'allegoria. Le Romain est entouré de signes du divin, qu'il interprète en ayant recours au significare. Il organise, il donne un axe au regard, in medio. Le but d'une telle structuration en est le dépassement par la contemplation, le prospectus, la vision du lointain (SAURON 2009, p. 277-283).

Exemplaire, la célèbre frise de la Villa des Mystères à Pompéi est une invite rare chez les Grands du monde romain (fig. 5). La lecture centralisée qu'en fait Sauron (reprise chez SAURON, 2009, p. 105-128), suivi par Croisille (CROISIlle, 2005, p. 142-150), s'accompagne d'une exégèse typologique des figures disposées selon le principe cher à l'Antiquité de la narration continue. Nous sommes face à la dernière phase d'un rite d'initiation aux mystères dionysiaques, reliée symboliquement au démembrement de Dionysos, à sa résurrection et à son apothéose. Un intérieur d'exception se livre ainsi aux yeux des spectateurs d'aujourd'hui, qui ne font qu'effleurer les conditions historiques de sa conception. L'expression de l'imaginaire d'une certaine élite se fait palpable : est-il peuplé de rêves de dépaysement et de grandeur ou plutôt riche de sens ? Si le discours parfois polémique tonifie le propos de Sauron, c'est la distance critique qui finalement prime à la lecture de ses ouvrages, la rigueur de l'analyse, l'usage de tous les éléments disponibles pour approcher un tant soi peu de 
l'intérieur l'histoire romaine à travers la personnalité des hommes qui l'ont vécue et l'art qu'ils ont promulgué.

Pour mener une telle réflexion, une préoccupation se fait jour qui relie la plupart des ouvrages présentés : l'usage en quantité d'une illustration de qualité ${ }^{9}$. Ainsi s'instaure un aller et retour parlant entre texte et image, dépassant l'illustration du discours ou l'explication d'œuvre, dans une interaction signifiante. Un pas supplémentaire reste à faire qui n'a été que partiellement franchi par Baldassarre et ses collègues : la prise en compte des témoins provinciaux attribuables aux mêmes périodes et plus tardifs que les exemples campaniens ou ceux de la région de Rome. C'est à ce taux-là que s'offrira enfin une vision de la variété de l'art romain, riche d'influences locales pour un discours général. Rappelons-nous donc enfin la réflexion de Pline l'Ancien dans son Histoire Naturelle (XXXVI, 101) : monuments et ouvres d'art sont des lieux de mémoire ; ils conservent le souvenir de leurs origines.

1. Les actes paraîtront au plus tard en 2013, selon le rythme des colloques de l'AIPMA.

2. Alix Barbet, La peinture murale en Gaule romaine, Paris, 2008 , et La peinture murale romaine : les styles décoratifs pompéiens, Paris, 2009.

3. Stendhal, Rome, Naples et Florence en 1817, Paris, 1817, cité dans Robert Étienne, Pompéi, la cité ensevelie, Paris, 1987, p. 158.

4. Ida Baldassarre éd., Pompei: pitture e mosaici, 11 vol., (Enciclopedia dell'Arte Antica, Classica e Orientale), Rome, 19901999.

5. Claudia Liedtke, Nebenraumdekorationen des 2. und 3. Jahrhunderts in Italien, (Jahrbuch des Deutschen Archäologischen Instituts, Ergänzungsheft, 31), Berlin/New York, 2003.

6. Karl Schefold, La peinture pompéienne : essai sur l'évolution de sa signification, Bruxelles, 1972 [éd. orig. : Pompejanische Malerei, Sinn und Ideengeschichte, Bâle, 1952].

7. Hamdallah BÉARAT, Michel FuCHS, Marino MAGGETTI, Daniel PAUNIER (ed.), Roman Wall Painting: Materials, Techniques, Analysis and Conservation, Proceedings of the International Workshop Fribourg 7-9 March 1996, Fribourg, 1997.

8. Agnès Rouveret dans BALDASSARRE et al., 2006, p. 9499, 146-147, 239-241. Voir aussi Agnès Rouveret, "Pictos ediscere mundos". Perception et imaginaire du paysage dans la peinture hellénistique et romaine, dans Ktema, 29, 2004, p. 325-344.
9. Les études réunies sous le titre Couleurs et matières dans l'Antiquité ne suivent pas le même but que les autres ouvrages : issues d'un colloque universitaire centré sur la part du texte à la réflexion sur l'image, elles réduisent les illustrations au strict nécessaire.

Michel E. Fuchs, Université de Lausanne Michel.Fuchs@unil.ch 